\title{
Investigating Stresses Developed during Mechanical Forming of Steel through Finite Element Analysis
}

\author{
S.A. Akinlabi ${ }^{1, a^{*}}$, O.S. Fatoba ${ }^{2, b}$ and E.T. Akinlabi ${ }^{2, c}$ \\ ${ }^{1}$ Department of Mechanical and Industrial Engineering Technology, University of Johannesburg, \\ South Africa \\ ${ }^{2}$ Department of Mechanical Engineering Science, University of Johannesburg, South Africa \\ astephenakinlabi@gmail.com, bdrfatobameni@gmail.com, ${ }^{c}$ etakinlabi@uj.ac.za
}

Keywords: Finite Element Analysis, Stresses and Structural Integrity

\begin{abstract}
Stresses majorly affect the mechanical properties of materials. However, structural failures are often caused by the combined effect of residual stresses and applied stresses. It is practically impossible for a manufactured component to be entirely free of residual stresses because these stresses developed during the manufacturing process and certain amount remain in the component even after the process is completed. This study reports the findings of the investigation into the developed stresses during mechanical forming of the steel sheet. The result revealed that the Von Mises stresses developed, increases during the forming process. Also, the original tensile stresses in the material changed to compressive stresses along the inner radius as the punch strokes increases. Lastly, it was observed that the locked in stresses in the material after the process were tensile in nature and such are not beneficiary to the structural integrity of the manufactured component even though an average value of $0.057540 \mathrm{MPa}$ was recorded for this study at the bend radius, distance away from the neutral plane.
\end{abstract}

\section{Introduction}

Finite element analysis has been a useful tool in research and engineering in particular for optimising and validating experimental procure and results. It has also been viewed as a tool deployed into design projects to save time and money through preliminary work using finite element analysis to establish the possible parameters and results. The experimental results are further validated with the finite element analysis results. Several types of research have deployed FEM to several manufacturing processes today. The demand in the manufacturing industry to design complex shapes to meet the new realities is the increase in the twenty-first century. The automotive industry, in particular, requires that the body shape of the cars be subjected to multiple and complicated loadings but also a strain rate that ranges between $0.1 \mathrm{~s}^{-1}$ and $100 \mathrm{~s}^{-1}$. Metal sheet forming under this conditions are often characterised with shape and surface distortions, spring back, wrinkling and induced stresses. Finite element methods are a useful tool and approached to manage the defect by conducting finite element analysis to estimate the expected results [1-3].

Furthermore, it is known that the material properties of steel sheets are influenced by intermediate strain rates among other things [4]. At intermediate strain rates higher than a strain rate of tens per second, materials experience the effect of the inertia and the stress wave propagation, consequently greatly altering the material properties. Various techniques, such as the mechanical method [5] and the drop weight method [6], have been tried to measure the material properties at intermediate strain rates.

Residual stresses would unavoidably be induced in the sheet material in the most manufacturing process, but their magnitude would depend on whether the sheet is produced by 
cold or hot working conditions. Rossini et al. [7] reported that residual stresses originated from some sources, which are introduced during manufacturing or in-service loading. These stresses can be present even in the unprocessed raw material. Furthermore, they suggested that the origin of residual stresses could be classified according to differential plastic flow, differential cooling rates, and phase transformations with volume changes.

According to the Welding Institute (TWI) [8], these stresses as being caused by incompatible internal permanent strains that may be generated at every stage of the life cycle of a component; from the original production to the final disposal. Withers [9] on the other hand, considered residual stresses to be developed because of misfits (incompatibilities) between different regions of the material, sample or assembly. Flaman [10] classified residual stresses according to how it developed. Kannatey-Asibu [11] defined residual stresses as stresses that continue to exist in a material even when no external forces are acting on it. He believed that such stress could be produced in some ways, some of which are during welding, grinding, bending, forming, and heat treatment processes. The effect can be detrimental when it reduces the tolerance of the material to an externally applied force. In this paper, the authors' present an investigation into the stresses developed during mechanical forming of steel through Finite Element Analysis.

\section{Mechanical Forming Process}

The process of bending result in both tension and compression in the sheet [12], with the outer radius and the inner radius of the sheet, undergo tension and compression respectively. The schematic of a bending process is shown in Fig. 1. Some of the standard parameters in a bending process such as bend angle, neutral axis, bend allowance and set back are shown.

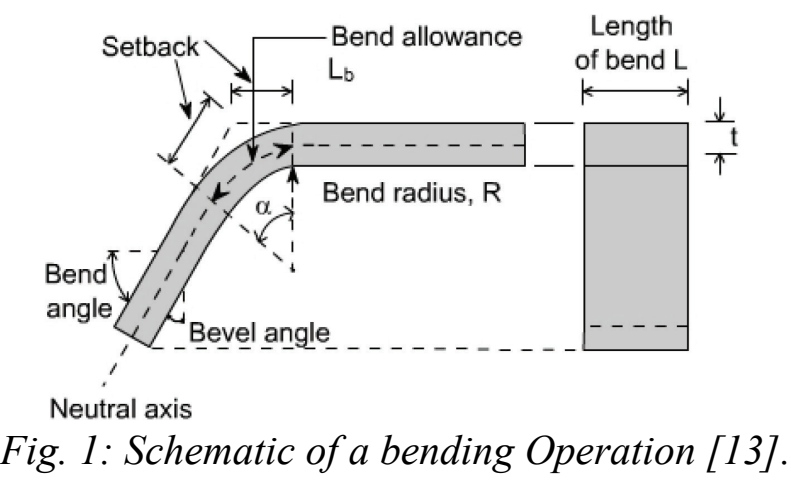

This phenomenon may be related to the bend allowance and bend deduction. However, it is important to state that due to the plastic deformation, there is residual stresses and strains after the forming process. These residual stresses consequently brings about elastic recovery in the material often called spring back which causes shape error in the final fabricated part.

\section{Finite Element Analysis}

Finite element analysis remain a tool that would continue to be relevant in all spheres of endeavour. This was applied to sheet forming process to evaluate the response and the mechanical properties of the material under loading. Marc software, 2015 version was employed for the analysis. The analysis is static but with elastic - plastic material characteristics.

\section{Assumptions}

The following assumptions were made:

- Analysis is static and assumed to be a simply supported;

- The hardening rule was based on Isotropic Model;

- The yield criterion was based on Von Mises; 
- The Poisson ratio was assumed to be 0.3 ;

- The punch has initial contact with the sheet;

- A constant time stepping approach was employed with $0.01 \mathrm{~s}$ and $0.025 \mathrm{~s}$ for loading and unloading respectively;

- Convergence tolerance of $0.1 \%$;

\section{Boundary Condition}

- Constrain movements of sets of nodes along the X-axis at the contact of the punch and the plate;

The geometry of the steel was defined in Mentat Marc, a plane strain element was used, and the material properties and boundary conditions were set up. The schematic diagram of the mechanical forming setup showing both the deformable and rigid bodies and the resulting deformation at five-punch stroke are shown in Fig. 2.

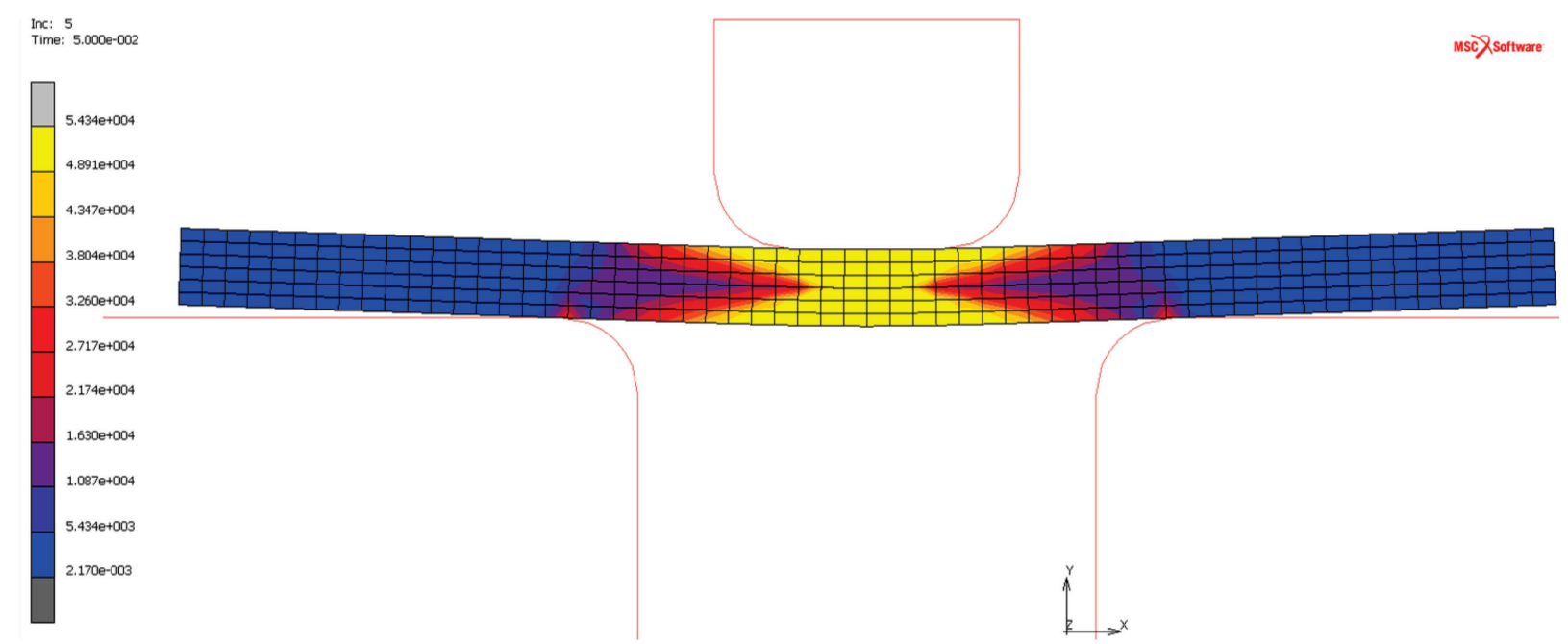

Fig. 2: Schematic of Mechanical Forming process setup and Stress Distribution at fifth stroke.

\section{Result and Discussion}

The finite element of the static analysis with elastic-plastic behaviour was conducted. In this study, the development of Von Mises stress was investigated and reported. The induced stresses into the sheet at the repeated punch stroke cycle of five were mainly tensile as displayed on the contour plot. It was also noted that the cloud of induced tensile stresses mainly concentrated around the contact area between the punch and the sheet, this was in agreement with the findings of both Rossini et al. [7] and Kannatey-Asibu [11].

The mechanical process of this nature in manufacturing is characterised by both tension and compression in the process, whereby consequently induce stresses some of which are naturally relieved during or at the end of the process while the rest remain within the manufactured or processed material. This phenomenon has been demonstrated through this study.

It was also noted that the bend angle increased from 9.8 degrees to 52.3 degrees at the fifth strokes, this is very significant and as such advised to be monitored during the manufacturing process.

The distribution of the sets of measured Von Mises stresses with increasing number of strokes is shown in Fig. 3. The plotted stress distributions show a progressive increase in both the inner and outside radius as the punch stroke cycle increases. 


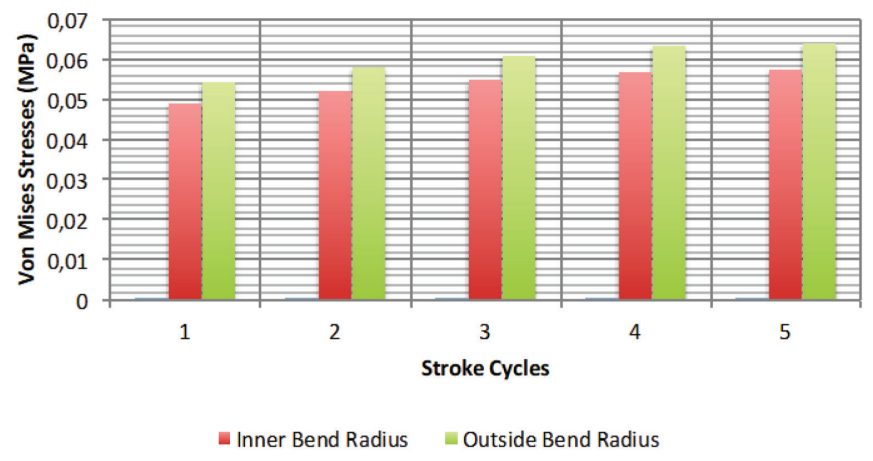

Fig. 3: Distribution of the Von Mises Stress.

Further analysis of the developed stresses during the mechanical forming process revealed the evolutional changes that characterised the forming process, which is critical to establish the stroke threshold that will give tolerable stresses considering the structural integrity that may be desired for the formed component. Four developed Von Mises Stresses were measured but only two at stroke cycles two and five were shown in Figs. 4 and 5. The process of sheet forming employs tensile force in the plane of the transverse direction of the sheet this consequently induces multi-stresses condition in the sample causing the bending of the sheet. This process entails both tension and compression in the sheet due to the punch impact on the sheet.

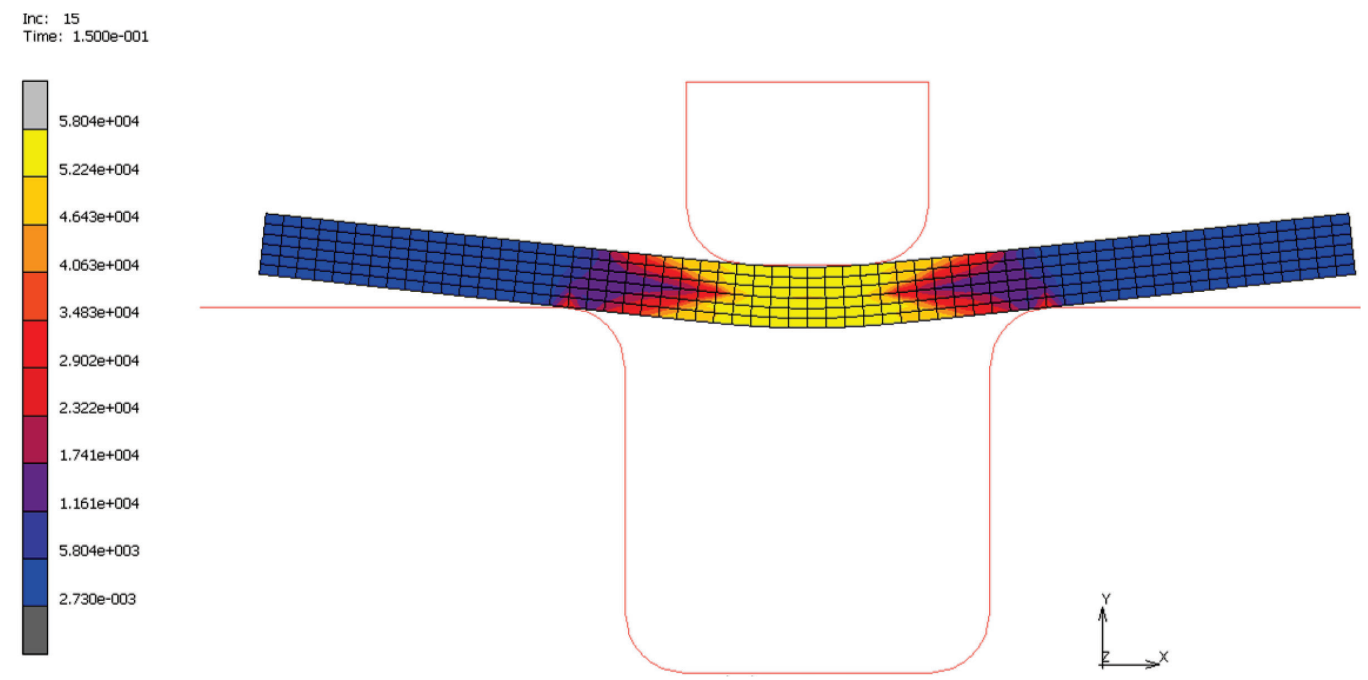

Fig. 4 : Equivalent Von Mises Stresses measured at stroke cycles of two.

It is observed that the impact of the stroke cycles in the forming of the sheet was significant. The first thing worth noting was the tensile nature of the developed stress along both the inner and outside bend radius but less compressive along the inner bend radius. However, the trend observed in all the samples was that the developed stresses become less tensile and more compressive along the neutral axis of the sheet as the bending process progresses, even though experimentally stresses at the neutral axis tend towards zero. This points to the importance of the neutral axis in sheet forming operation. The neutral axis is the plane separating the inner bend radius from the outer bend radius. As the number of the cycle stroke increases, the dimensions of the thickness of the neutral axis increases to as high as $1.5 \mathrm{~mm}$, such an increase was observed to be very significant.

Hence, effective sheet forming operation controls and ensures the stress locked around the neutral axis is within tolerable values otherwise it can be catastrophic. This further confirms the 
significant role of the punch stroke during a sheet forming operations. Another important observation worthy of highlighting is the formation of cup-like configuration around the bend area. This cup-like shape is a mirror of the punch geometry and as such this will be an excellent tool to control the developed stresses through the design of the punch geometry and material.

The relaxation during the first unloading during the forming process is shown in Fig. 6. During the unloading of the punch at the end of the complete cycle, even though the nature of the stress remains tensile and compressive, a significant stress relaxation was observed. The magnitude of the stress around the neutral axis during the last loading stroke was $0.057540 \mathrm{MPa}$ while at the first unloading stroke, the magnitude of the stress along the neutral axis was $0.031650 \mathrm{MPa}$. This relaxation phenomenon is often called elastic recovery.
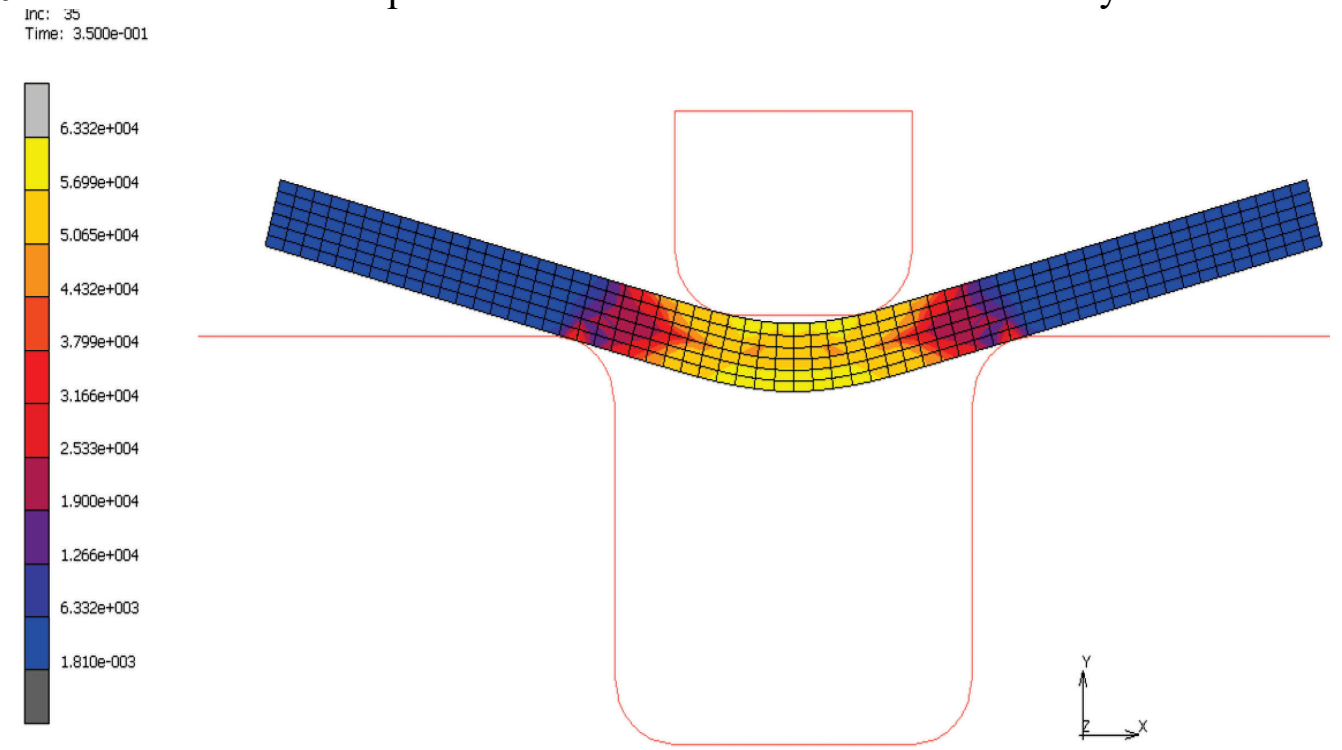

Fig. 5: Equivalent Von Mises Stresses measured at stroke cycles of five.
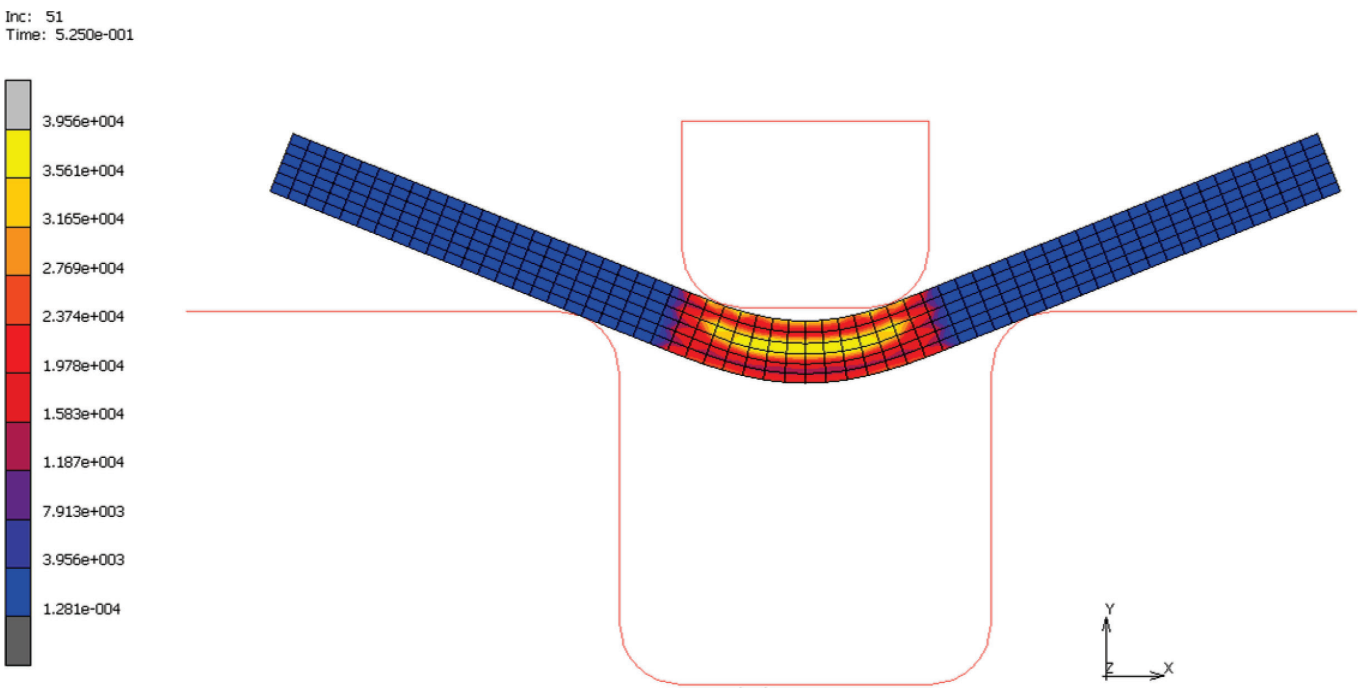

Fig. 6: Relaxation at the first unloading during the forming Process.

\section{Conclusion}

The finite element analysis of the mechanical forming of sheet steel was conducted and completed. The following conclusions can be drawn:

- Tensile and compressive stresses are developed during the mechanical forming process, and it increases as the punch stroke increases. 
- The stresses along the inner and outside radius are tensile while stresses close and around the neutral axis are more compressive.

- The stress concentration close to the neutral axis increases as the punch strokes increases.

- The stress distributions form a cup-like configuration, which is a reflection of the geometry of the punch.

- An elastic recovery of $0.025890 \mathrm{MPa}$ developed at the unloading of the punch.

\section{References}

[1] G. Joo, H. Huh and M.K. Choi, Tension/Compression hardening behaviors of auto-body Steel Sheets at Intermediate strain rates, Int. J. Mech. Sci. 108-109 (2016) 174-187. https://doi.org/10.1016/j.ijmecsci.2016.01.035

[2] H. Huh, J. H. Lim and S. H. Park, High speed tensile test of steel sheets for the stress - strain curve at the intermediate strain rate, Int. J. Mech. Sci. 2009 10(2) 195-204. https://doi.org/10.1007/s12239-009-0023-3

[3] M.A. Meyer, Dynamic behavior of materials, John Wiley \& Sons, N.Y, U.S.A, 1994. https://doi.org/10.1002/9780470172278

[4] D.R. Ambur, C.B. Prasad and W.A. Waters Jr., A dropped-weight apparatus for Low Speed impact testing of composite structures, Exp. Mech. 35 (1995) 77-82. https://doi.org/10.1007/BF02325839

[5] N.S. Rossini, M. Dassisti, K.Y. Benyounis and A.G. Olabi. Methods of measuring residual stresses in Samples, Material and Design, 35 (2012) 572-588. https://doi.org/10.1016/j.matdes.2011.08.022

[6] TWI Knowledge Summary, 2015, www.twico.uk/j32/protected/band_3/ksrh1001.html [Accessed February 2017]

[7] P.J. Withers, Residual stress and its role in failure, Report of progress in Physics 70 (2007) 2211-2264. https://doi.org/10.1088/0034-4885/70/12/R04

[8] M.T. Flaman, Investigation of ultra-high speed drilling for residual stress measurements by the Centre-hole method, Exp. Mech. 22 (1) (1982) 26-30. https://doi.org/10.1007/BF02325700

[9] E. Kannatey-Asibu, Principle of Laser Materials Processing, John Wiley \& sons, Inc., N.Y., 2009. https://doi.org/10.1002/9780470459300

[10] Sheet Metal Processes, http://nptel.ac.in/courses/112107144/6 [Accessed March 2017].

[11] G.H. Bae and H. Huh, Tension/compression test of auto-body steel sheets with the variation of the pre-strain and the strain rate, In: Proc. 5th Int. Conf. on Computational Methods and Experiments in Materials Characterisation, Kos, Greece. 2011. https://doi.org/10.2495/MC110191

[12] S. Choudhry and J.K. Lee, Dynamic plane-strain finite element simulation of industrial sheet-metal forming processes, Int. J. Mech. Sci. 36 (1994) 189-207. https://doi.org/10.1016/0020-7403(94)90069-8

[13] H. Tze-Chi and C. Chan-Hung, A finite element analysis of sheet metal forming processes, J. Mater. Process. Technol. 54 (1995) 70-75. https://doi.org/10.1016/0924-0136(95)01922-7 